\title{
DEVELOPMENT OF THE ELECTROPHYSIOLOGICAL PROPERTIES OF Y-CELLS IN THE KITTEN'S MEDIAL INTERLAMINAR NUCLEUS ${ }^{1}$
}

\author{
JAMES R. WILSON, ${ }^{2}$ DAVID E. TESSIN, AND S. MURRAY SHERMAN \\ Department of Neurobiology and Behavior, State University of New York at Stony Brook, Stony Brook, New York 11794
}

Received September 8, 1981; Revised December 23, 1981; Accepted December 30, 1981

\begin{abstract}
Single unit, extracellular recordings were made in the medial interlaminar nucleus (MIN) of adult cats and of kittens at 2, 4, 6, 8, 12, and 16 weeks of age. MIN is part of the dorsal lateral geniculate nucleus, and nearly all of its recorded neurons are Y-cells in adult cats. We find that Y-cells in the MIN of younger kittens have long latencies to optic chiasm stimulation, large receptive fields without surrounds, low spatial and temporal resolutions, and nearly absent spontaneous activity. Responses to hand-held light stimuli are typically poor and inconsistent. Also, most of these immature Y-cells exhibit only linear response properties when tested with a counterphased modulated grating pattern. All of the above characteristics gradually develop to adult values over several months of the postnatal period. The percentage of cells with nonlinear response properties becomes adult-like near 8 weeks of age, while both spatial and temporal resolutions are still developing at 16 weeks of age. Latencies and receptive field center sizes achieve adult values at about 12 weeks of age. The immature properties of these neurons are similar to those recorded from abnormal cells in the area of the medial interlaminar nucleus innervated by the deprived eye of monocularly deprived adult cats. This indicates that the adult deprived Y-cells probably fail to develop and thus retain their immature properties.
\end{abstract}

The dorsal lateral geniculate nucleus of all carnivores and ungulates examined to date has a subdivision that has been termed the medial interlaminar nucleus or MIN (Thuma, 1928; Karamanlidis and Magras, 1972, 1974; Sanderson, 1974). In cats, this subdivision contains a nearly homogeneous population of Y-cells (Palmer et al., 1975; Mason, 1975; Kratz et al., 1978a, b; Dreher and Sefton, 1979; see also "Discussion"). Since MIN projects to cortical areas 18,19 , the lateral suprasylvian gyrus, and perhaps sparsely to area 17 (Garey and Powell, 1967; Niimi and Sprague, 1970; Rosenquist et al., 1974; Maciewicz, 1974, 1975; Gilbert and Kelly, 1975; LeVay and Ferster, 1977; Hollander and Vanegas, 1977; Geisert, 1980), it potentially has a direct influence on many areas of visual cortex. Like the laminated part of the lateral geniculate nucleus, MIN receives afferents from both the retina and cortex, is retinotopically organized, and has separate areas or laminae of termination for the afferents from each eye (Garey and Powell, 1968; Guillery, 1970;

\footnotetext{
'We thank Sally Gibson and Joan Sommermeyer for their expert technical assistance. This research was supported by United States Public Health Service Grant EY 03345.

${ }^{2}$ To whom correspondence should be addressed at Department of Neurobiology, State University of New York at Stony Brook, Stony Brook, NY 11794.
}

Sanderson, 1971; Updyke, 1975; Kratz et al., 1978a; Guillery et al., 1980). Because MIN is well defined anatomically and has essentially only one physiologically demonstrable cell type, it is particularly suitable for developmental studies. Such studies are of value when we wish to consider the mechanisms responsible for these developmental sequences and how such development can be altered under the influence of disease or abnormal environments.

Our major goal in this study was to define the electrophysiological properties of the Y-cell population of MIN in developing kittens. We addressed four main questions: (1) what are the characteristics of immature Y-cells? (2) How do these characteristics change with age? (3) When do they become mature? (4) How do the immature properties compare with those of Y-cells in cats reared to adulthood with monocular lid suture (cf., Kratz et al., 1978b)? The last question is part of a series of studies designed to determine how the environment interacts with the developing visual system (for recent reviews, see Movshon and Van Sluyters, 1981; Sherman and Spear, 1982).

\section{Materials and Methods}

Subjects. We recorded single neuron activity from 7 adult cats and 17 kittens ranging in postnatal age from 2 to 16 weeks. The kittens included 3 at 2 weeks of age, 2 
at 4 weeks, 5 at 6 weeks, 4 at 8 weeks, 2 at 12 weeks, and 1 at 16 weeks. These animals were obtained from our breeding colony. Each kitten was weighed before use. Although there was a range of weights for animals of the same age, we saw no correlation between this parameter and the maturity of the neurons studied.

Physiological preparation. Our overall electrophysiological procedures were similar to those previously described for adult cats (Kratz et al., 1978a; Lehmkuhle et al., 1980a) with some modifications for kittens. The animals were anesthetized with halothane (1 to $2 \%$ ) in a $1 /$ $1 \mathrm{~N}_{2} \mathrm{O} / \mathrm{O}_{2}$ mixture during surgery and afterward with a $70 \% / 30 \%$ mixture of $\mathrm{N}_{2} \mathrm{O} / \mathrm{O}_{2}$ alone for the 18-hr recording session. Procaine dissolved in peanut oil was applied to pressure points and wound margins, a venous cannulation for infusion of the paralytic (gallamine triethiodide), a tracheal tube for artificial respiration after paralysis, and craniotomies made for stimulating and recording electrodes. Contact lenses with 3-mm-diameter artificial pupils and of the proper radius for the curvature of the kitten's cornea (Thorn et al., 1976; Freeman, 1980) protected the eyes and placed the focal plane near $57 \mathrm{~cm}$. Refraction was verified with both streak retinoscopy and neuronal response properties (i.e., additional lenses were placed in front of the eyes when necessary to achieve maximum spatial resolution). Rectal temperature and heart rate were monitored continuously. For very young kittens, additional mechanical support was given to the head by cementing a flat plate to the posterior skull and attaching this plate to a rigid stereotaxic crossbar. Also for kittens, we incorporated 1 to $2 \% \mathrm{CO}_{2}$ into the $\mathrm{N}_{2} \mathrm{O} / \mathrm{O}_{2}$ gas mixture and increased the stroke rate of the respirator (Daniels et al., 1978). Bipolar stimulating electrodes, consisting of insulated metal except for $1 \mathrm{~mm}$ of the tip, were positioned across the optic chiasm. Their placement was guided by recording the potential evoked across them when a strobe flash was given to the eyes.

Recording procedures. Glass micropipettes (10 to 30 megohms impedance measured at $100 \mathrm{~Hz}$ and filled with $4 \mathrm{M} \mathrm{NaCl}$ ) were used to record from individual, well isolated neurons. Penetrations were made at $30^{\circ}$ to the vertical in the coronal plane so that the electrode tip traveled medially and ventrally. Because of this angle, cells in the $\mathrm{A}$ and $\mathrm{C}$ laminae were encountered prior to MIN, and they served three purposes. First, we could evaluate the general properties of immature neurons in the $\mathrm{A}$ and $\mathrm{C}$ laminae for comparison with those of MIN. Second, because the position of the consecutively plotted receptive fields slowly moved toward the vertical midline, we could judge from Sanderson's (1971) retinotopic maps whether we would encounter MIN during a given penetration. Finally, as soon as the receptive field progression reversed (i.e., moved away from instead of toward the vertical midline), we could be sure that we were in MIN. We limited our penetrations to the thicker, more ventral portion of MIN and thereby avoided the area of the geniculate “wing” (Guillery el al., 1980).

The receptive fields of these neurons were studied with small, hand-held spots of light on a frontal tangent screen and with gratings produced on an oscilloscope (see below). For tangent screen plotting, background illuminance was $0.6 \mathrm{~cd} / \mathrm{m}^{2}$ which is in the mesopic range of the cat (Hammond and James, 1971), and the stimulus illu- mination was about $1.0 \mathrm{log}$ unit above this. We measured the receptive field center positions relative to the optic disc center. Corrections were made for tangent screen skewing plus the change with age of the distance between the optic disc and the area centralis (Olson and Freeman, 1980). Despite the cloudy optics in the young kittens, we were always able to see the optic disc. Except for 2 of the 3 kittens at 2 weeks of age, we also were able to project the optic disc image onto the tangent screen to determine eye position (Fernald and Chase, 1971). For the 2 kittens from which optic disc plots could not be obtained, we calculated the receptive field eccentricities relative to the point on the tangent screen directly in front of the eyes.

A grating pattern was produced electronically on a Tektronix 608 oscilloscope located $57 \mathrm{~cm}$ in front of the eyes. We could adjust the spatial frequency, temporal frequency of counterphase modulation, spatial phase position, and contrast (up to 0.85 ) of this grating pattern. The spatial and temporal modulation were produced using either sinusoidal or square wave input patterns. Typically, $2 \mathrm{~Hz}$ was used as the counterphase modulation rate, but lower rates were used for very young neurons which would not respond to $2 \mathrm{~Hz}$. The maximum temporal frequency for these grating patterns was 25 to 30 $\mathrm{Hz}$, and this limited the range of our tests for this parameter. For each neuron, we also measured the spatial and temporal resolutions and assessed whether its response properties were linear or nonlinear. Spatial resolution is defined as the highest spatial frequency (cycles per degree) to which the cell responded using a counterphased grating pattern at our maximum contrast level. Similarly, temporal resolution is defined as the highest counterphase rate of the grating to which the neuron continued to respond. The cell was determined to be linear if it had a grating position (spatial phase) to which it did not respond (the "null" position) and if the response occurred at the frequency of the stimulus. Alternatively, a nonlinear cell responded to each pattern reversal such that two responses were obtained for each counterphase cycle, resulting in a response at twice the temporal frequency, and this frequency-doubled response was independent of spatial phase, at least at higher spatial frequencies (Enroth-Cugell and Robson, 1966; Hochstein and Shapley, 1976).

For qualitative assessment of responses, the spikes were observed on an oscilloscope and monitored over an audio speaker. Peristimulus time (average response) histograms were taken in many cases to verify or quantify the responses of a cell to the stimulus pattern. These histograms were particularly useful when the responses of the cell were inconsistent or weak. These methods provided reasonable estimates of the temporal and spatial resolutions of each cell at 0.85 contrast. We tested each cell's nonlinear summation properties (which we shall call the doubling response) close to its maximum spatial resolution and at all spatial phase positions (Hochstein and Shapley, 1976; Lehmkuhle et al., 1980).

At the end of the experiment, the animals were anesthetized deeply with sodium pentobarbital and perfused through the heart. The brains were removed, and, in several cases, the electrode tracks were observed to pass through the middle portion of MIN. These cases confirmed that our assumption of MIN recording based on 
retinotopic considerations was correct (see also Kratz et al., 1978a). However, because we did not reconstruct most of the electrode tracks, we did not consider a neuron to be in MIN unless there was a progression of receptive fields toward the vertical midline followed by a clear jump of the receptive field position away from the midline. This progression indicates passage through the $\mathrm{A}$ and $C$ laminae, and a jump in position away from the vertical meridian is an unambiguous indication of passage of the electrode tip into MIN. Thus, our data for MIN include only a few cells which had receptive fields close to the vertical meridian (less than $3^{\circ}$ ). The mean eccentricity for our MIN cells is approximately $20^{\circ}$ for every age group except one: at 4 weeks of age, the average eccentricity is about $40^{\circ}$.

\section{Results \\ Adult $M I N$}

We collected data from 7 adult animals to establish normal adult properties for MIN cells, and these data confirmed and extended several previous reports (Palmer et al., 1975; Mason, 1975; Kratz et al., 1978a, b; Dreher and Sefton, 1979). We classified 26 out of 29 cells recorded in MIN as Y-cells (90\%). These 26 neurons exhibited short latencies to optic chiasm stimulation (average, 1.3 $\mathrm{msec}$ ), had relatively large receptive fields (average, $2.1^{\circ}$ ), were excited by large, rapidly moving discs (greater than $200^{\circ} / \mathrm{sec}$ ) of either contrast, and had large amplitude doubling responses. The doubling responses became audibly phase independent, on average, at a spatial frequency of 0.3 to 0.4 cycle/deg. At lower spatial frequencies, a doubling response was heard only at the spatial phase where the fundamental response was not dominating-the "null" position of the fundamental response. We also have found that adult MIN neurons have contrast sensitivity curves (Fig. 1) similar to those of the Y. cells in the A laminae (Lehmkuhle et al., 1980a). The peak contrast sensitivities, however, were higher in our MIN neurons (average, 77) than were those reported by Lehmkuhle et al. (1980a; average, 32). Furthermore, the mean spatial resolution from our MIN sample was lower (average, 1.9 cycles $/ \mathrm{deg}$ ) than the $3.0 \mathrm{cycles} / \mathrm{deg}$ reported previously for A laminae $Y$-cells (Lehmkuhle et al., 1980a). These results remain tentative (see "Discussion"), although our own qualitative comparison of A laminae $\mathrm{Y}$-cell responses with those of MIN Y-cells supports this conclusion.

The 3 cells which were not $Y$-cells were all recorded in 1 animal from penetrations in MIN which yielded mostly Y-cells. Two of the 3 had "null" positions and no frequency doubling. They were located 7 to $10^{\circ}$ lateral to the vertical meridian. One had a small receptive field $\left(0.4^{\circ}\right.$ in diameter) and a latency of $1.5 \mathrm{msec}$ and was classified as an X-cell. The second cell had a $1.5^{\circ}$ center diameter, a 1.4-msec latency, and a low spatial resolution ( 0.5 cycle/deg). We did not classify this cell. We were unable to obtain a latency for the third cell and it did not respond at all to the grating. This cell was possibly a Wcell (Wilson et al., 1976; Stanford et al., 1981; Sur and Sherman, 1982a).

Finally, the average receptive field center diameter of adult MIN neurons increased with visual field eccentric-

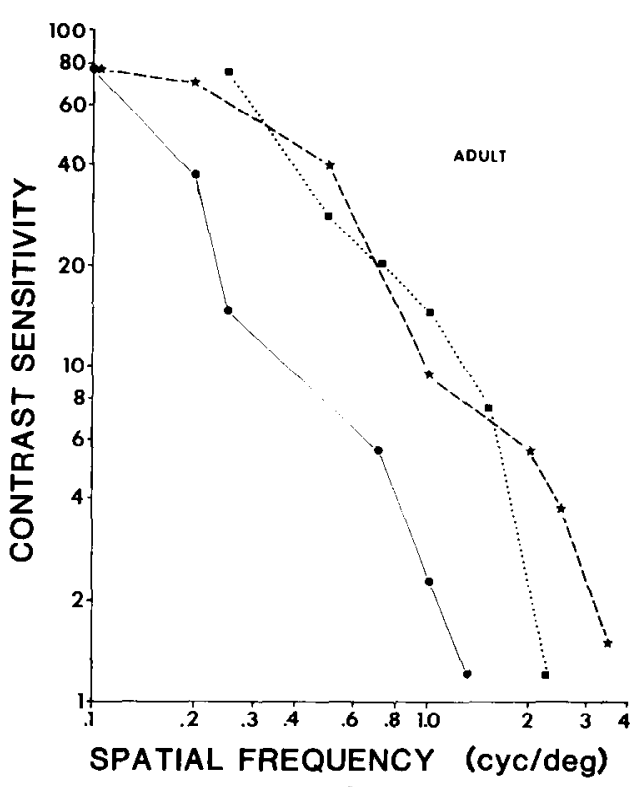

Figure 1. Spatial contrast sensitivity functions for 3 MIN Ycells recorded in adult cats. The contrast sensitivity is defined as the inverse of the lowest contrast necessary to excite the cell. The linear and nonlinear components were not distinguished for these functions.

ity. The slope of the linear regression line which fit this data was $0.058^{\circ}$ in diameter/deg of eccentricity and the $y$ intercept occurred at $1.1^{\circ}$. The correlation coefficient was 0.46 .

\section{Kitten $M I N$}

\section{Qualitative analysis}

The electrophysiological properties of neurons in MIN of younger kittens were difficult to assess due to their inconsistency and poor responsiveness. We usually were able to plot a receptive field center even for the youngest kittens ( 2 weeks), but 2 out of 17 responded in an on/off fashion to the smallest light spots that evoked activity. Excitatory surround responses were not seen for MIN neurons before 6 weeks of age. For most of the very immature MIN neurons, the light spots were much larger (by about 2 to 3 times) than the size necessary to stimulate an adult $\mathrm{Y}$-cell, and the neurons did not always respond to every stimulus presentation.

The inconsistent response of one of these neurons to a counterphased, square wave grating is demonstrated in Figure 2. No doubling response was observed for this neuron for the first few minutes of testing irrespective of spatial frequency or phase of the grating. After we obtained several successive histograms of these responses (Fig. 2, $A, B$, and $C$ ), we began collecting another histogram when we suddenly observed the cell's response begin to modulate at twice the temporal frequency. This histogram is shown in Figure 2D. Eye movements relative to the grating cannot account for this dramatic change since we had tested at all spatial phase positions previously, and, in addition, no movement of the receptive field was evident on the tangent screen. While it was fortuitous in this case that the temporal lability occurred while we were obtaining histograms, this was not an unusual observation. Indeed, a number of other imma- 


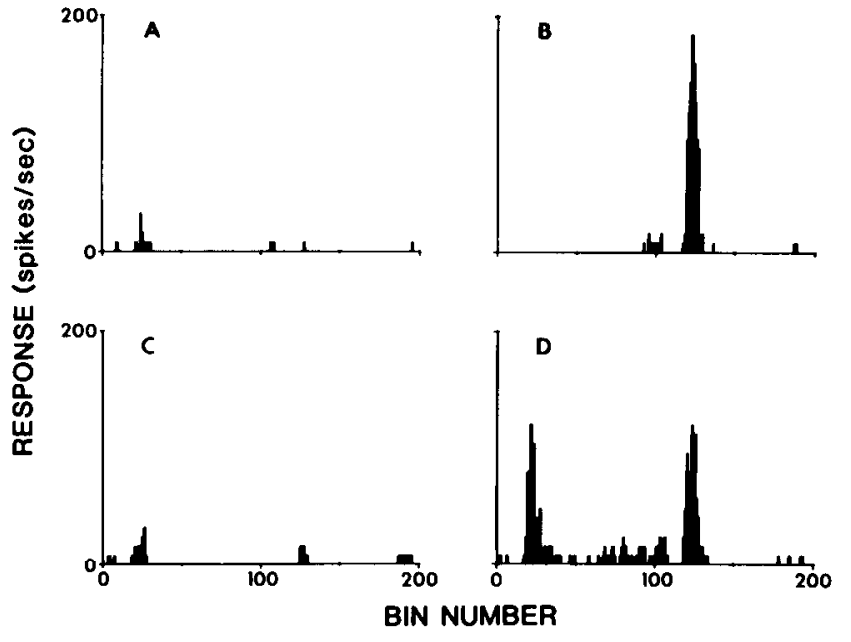

Figure 2. Peristimulus time (average response) histograms for an immature MIN Y-cell from a 16-week-old kitten. These demonstrate the variability of the doubling response. Each histogram represents the average response over 50 stimulus presentations (100 reversals of the grating pattern; total time per bin, $125 \mathrm{msec}(2.5 \mathrm{msec} / \mathrm{bin} \times 50$ trials $))$ at a spatial frequency of 0.3 cycle/deg and a temporal frequency of $2 \mathrm{~Hz}$ using square wave modulation and 0.85 contrast. The spatial resolution for this cell was $0.4 \mathrm{cycle} / \mathrm{deg}$ initially. The grating reversals occur at bins 0 and 100 (bin 200 is thus equivalent to bin 0 ). $A$, Response for spatial phase position of the grating at the "null" position for the fundamental response. $B$, Response to the same stimulus as in $A$ but shifted $90^{\circ}$ in spatial phase and recorded $1 \mathrm{~min}$ later. $C$, Another histogram at the same phase position as in $A$ recorded about $2 \mathrm{~min}$ after $B . D$, Repeat of $C$ using exactly the same parameters and taken 1 min later. The cell now shows a clear, strong doubling response.

ture MIN cells and cells of the A laminae (S. Mangel, J. R. Wilson, and S. M. Sherman, manuscript in preparation) also exhibited temporally labile nonlinear response components similar to the one illustrated. When the nonlinear response became apparent for these MIN neurons, their spatial resolutions became higher and the responses were phase independent as in mature Y-cells (Hochstein and Shapley, 1976).

Even with the poor and inconsistent responses, it was clear to us that neurons in these young kittens had the following characteristics: (1) large receptive field center sizes and no surround responses before 6 weeks of age that we could detect using hand-held light spots or annuli; (2) long latencies to optic chiasm stimulation; (3) low temporal and spatial resolutions; (4) poor responses to visual stimuli; (5) poor, inconsistent, or nonexistent doubling responses to counterphased modulated gratings; and (6) low spontaneous activities. Many immature neurons in the $\mathrm{A}$ and $\mathrm{C}$ laminae also had most or all of these properties.

\section{Quantitative analysis}

We quantitatively examined four properties of developing MIN neurons: (1) latency to optic chiasm stimulation, (2) receptive field center diameter, (3) spatial resolution (typically at a $2-\mathrm{Hz}$ counterphase rate), and (4) temporal resolution at or near the most sensitive spatial frequency for the neuron. Nonparametric, oneway analysis of variance tests (Kruskal-Wallis; see Siegel,
1956) showed each of these variables to change significantly $(p<0.001)$ over the postnatal period. The presence or absence of an audible doubling response also was determined; cells without this doubling had a "null" position and were, therefore, classified as linear (see "Materials and Methods"). The percentage of MIN cells exhibiting such doubling increased postnatally $(p<0.001$ on a $\chi^{2}$ test). A few contrast sensitivity functions were obtained, but because of the poor responses of the immature neurons, we found this to be a long and tedious task and we frequently lost the neurons during the process. Some of the few examples that we collected are shown in Figure 3. It should be noted that the maximum contrast sensitivities of kittens as young as 6 weeks of age are comparable to those of adults (cf., Fig. 1).

Latency. Figure 4 illustrates for MIN neurons the change with age of the mean latency to optic chiasm stimulation. The latencies were measured from the start of the stimulus artifact to the foot of the action potential and were taken as the middle of the range of values. The latency range at 2 weeks of age is 4 to $20 \mathrm{msec}$ and the average is 12 msec. Furthermore, the latency ranges for individual neurons were more variable at the younger ages than they were in the more mature kittens. The mean latency rapidly decreases with age and reaches an asymptote near 12 weeks of age at a value of $1.3 \mathrm{msec}$.

Receptive field center size. The average receptive field center size (expressed as the diameter of these circular receptive fields) decreases with age as shown in Figure 5. This decrease with time is fairly monotonic and reaches adult-like values at 12 to 16 weeks of age. The decrease is approximately 3 -fold between 2 and 16 weeks of age. The average values of the sample drawn from the $A$ laminae, which are biased toward the more mature Xlike cells, also are shown in this figure. Since the receptive

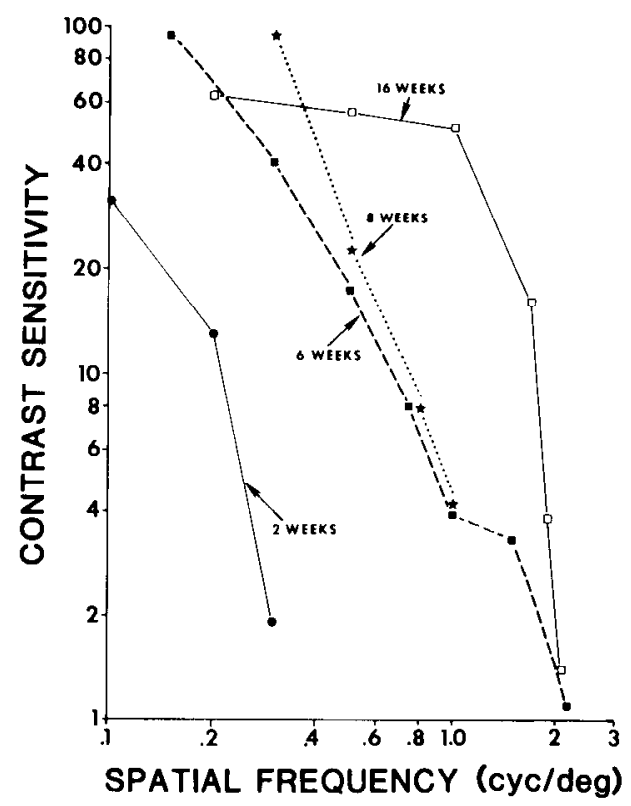

Figure 3. Contrast sensitivity functions for 4 neurons recorded in MIN of 4 different kittens at the ages shown. Note that the maximum sensitivities of the 3 cells from kittens 6 weeks and older are comparable to those of the adult neurons plotted in Figure 1. Other conventions are as in Figure 1. 


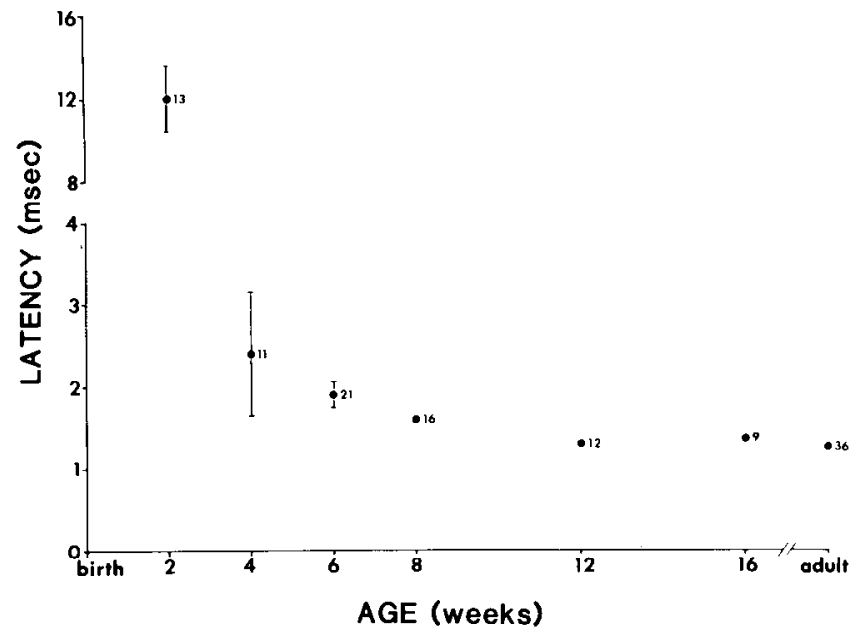

Figure 4. Average latency values to optic chiasm stimulation for neurons in MIN. The abscissa indicates the ages of the kittens from which the cells used to derive the averages were recorded. Standard error bars are shown for the means when they extend beyond the radius of the solid circles. Note the very large change in average latency between 2-week and 4week-old kittens and the vertical scale change between these ages. Next to each average point is the number of neurons used to derive the value of that point.

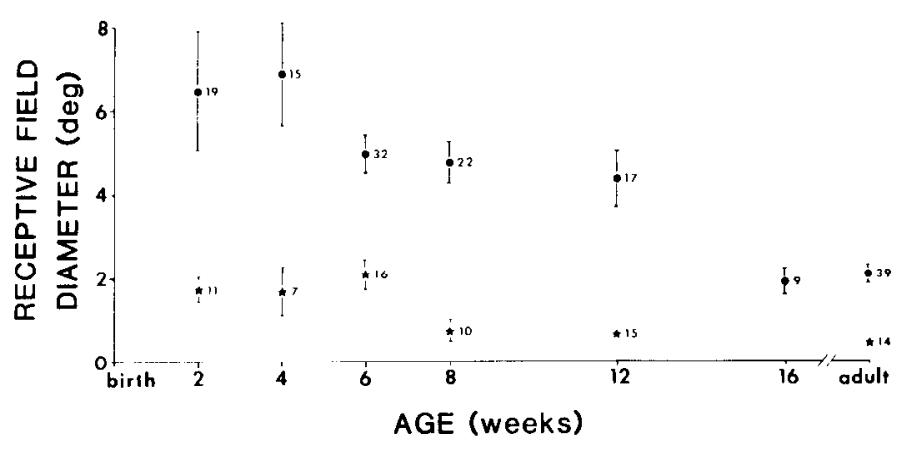

Figure 5. Average receptive field center sizes as a function of age in MIN of kittens. Since the fields were essentially all circular, we used their diameter (in terms of the visual angle subtended at the eye) as the size measure. The solid circles represent the data from MIN neurons. The stars represent the data from neurons in the A laminae. 'The A laminae data are highly biased toward the more mature cells in this area; i.e., these cells were nearly adult-like in their responsiveness, consistency, and receptive field properties. They are shown only to emphasize that small receptive field centers were seen commonly and optics did not play a major role in determining the larger size of MIN receptive field centers. Other conventions are as in Figure 4.

field center sizes of these A laminae cells were much smaller than those of MIN neurons at all ages, we feel confident that poor optics was not a major factor in determining the sizes for receptive fields of MIN neurons. However, the physical size of the eye as it develops from 2 weeks to adulthood probably does play a significant role in the change of receptive field size with age (Olson and Freeman, 1980; see "Discussion").

Spatial resolution. The average spatial resolution of neurons in MIN at different ages is shown in Figure 6. There is a monotonic increase of mean spatial resolution with increasing age, from 0.3 cycle $/ \mathrm{deg}$ at 2 weeks of age to 1.3 cycles/deg at 16 weeks of age. Even at 2 weeks of age, there were neurons in MIN or the A laminae which had spatial resolutions of 0.8 cycle/deg or higher, showing again that poor optics did not limit spatial resolution of the MIN neurons in young kittens. Since the average adult spatial resolution was found to be 1.9 cycles/deg, MIN neurons may not reach maturity for this property by 16 weeks of age. This also implies that the nonlinear response-which determines spatial resolution for mature Y-cells-is not yet fully developed at this age. We gathered insufficient spatial resolution data from neurons in the A laminae to make a useful comparison with the MIN data.

Temporal resolution. The average temporal resolution of MIN neurons to a counterphased modulated grating is illustrated as a function of age in Figure 7. The temporal resolution was always measured at a low spatial frequency $(\sim 0.1 \mathrm{cycle} / \mathrm{deg})$ because these cells are most sensitive to such low spatial frequencies (cf., Figs. 1 and 3 ). Our measurements of temporal resolution indicate considerable variability for each age. However, nearly all (8 of 9) of the adult neurons clearly responded at the

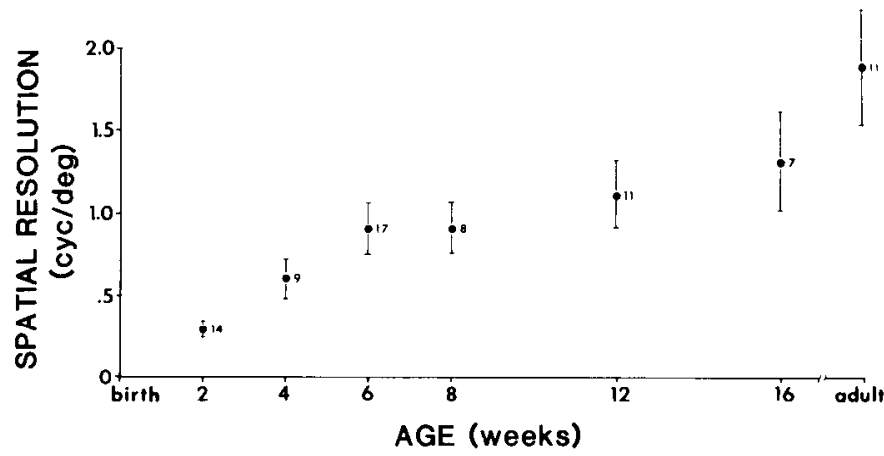

Figure 6. Average spatial resolution of MIN neurons at various ages. The resolutions were determined at 0.85 contrast by the response component which was highest (i.e., either the fundamental, particularly for the younger kittens, or the doubling response). Other conventions are as in Figure 4.

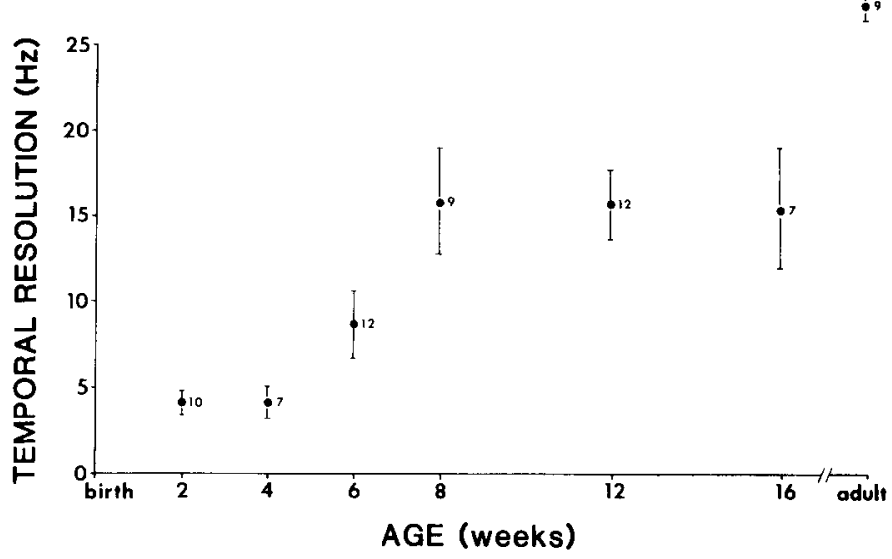

Figure 7. Average temporal resolution of MIN neurons of kittens at different ages. The adult value should be viewed as a minimum since our stimulus was incapable of going higher than 25 to $30 \mathrm{~Hz}$ and most MIN neurons in these adults easily reached this limit. Other conventions are as in Figure 4. 


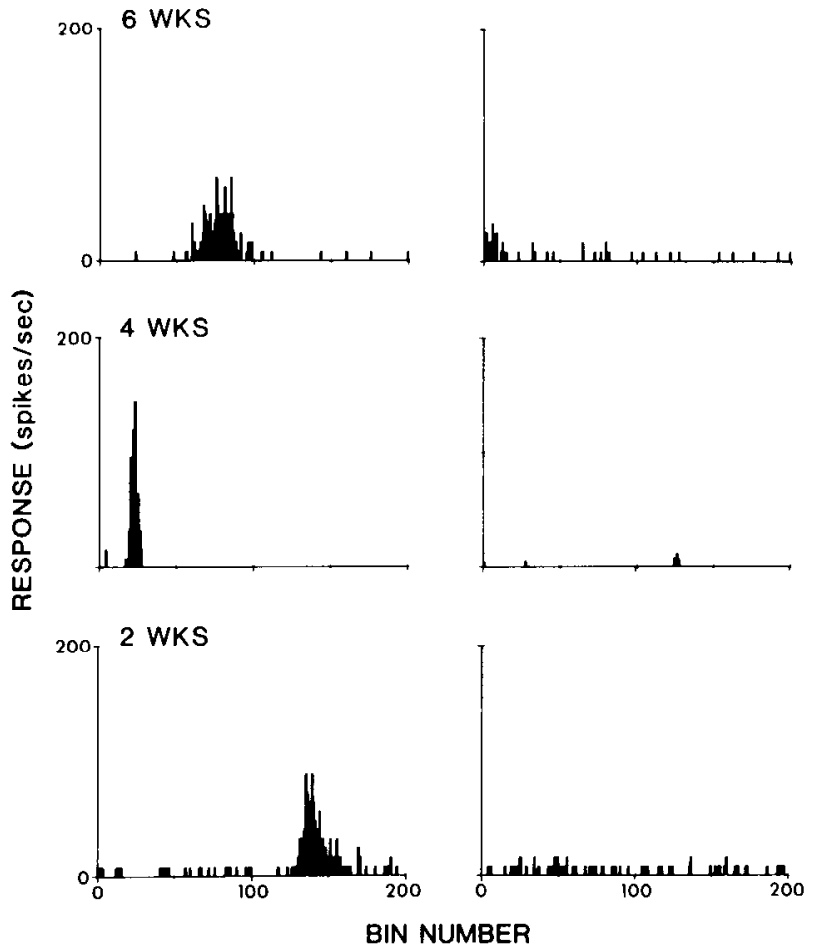

Figure 8. Peristimulus time histograms for 3 MIN neurons at the ages shown to demonstrate their lack of a doubling response. The left column shows the fundamental response taken as the $0^{\circ}$ phase position. The right column shows the lack of a response recorded with the grating pattern at a $90^{\circ}$ phase position. Otherwise, the stimulus parameters were identical for each pair and were for the $2-, 4-$, and 6 -week neurons, respectively: trials, 30,50 , and 50 ; spatial and temporal modulation waveform, square, sine, and square; spatial frequency, $0.075,0.2$, and 0.1 cycle/deg; temporal frequency, $1.0,2.0$, and $2.0 \mathrm{~Hz}$; contrast, $0.85,0.85$, and 0.85 . The spatial frequencies used for these tests, while quite low, nevertheless were close to the resolution of each cell.

limit of our visual stimulator ( 25 to $30 \mathrm{~Hz})$, while even at 16 weeks, many MIN neurons (4 of 7) were not capable of this resolution. The trend is much like that for spatial resolution, with temporal resolution, on average, failing to reach the adult value by 16 weeks of age.

Nonlinear responses. A prominent feature of $\mathrm{Y}$-cells is the doubling response component that dominates at higher spatial frequencies and is independent of spatial phase (Hochstein and Shapley, 1976). Such nonlinear responses were absent in many immature MIN cells even though the fundamental responses were clearly present (Fig. 8). We also noted that 2 of the 5 neurons with a doubling response in kittens 2 weeks of age had on/off center responses in contrast to adult Y-cells which never display such a property. Figure 9 further shows how the doubling responses are more prominent in peristimulus time histograms for older kittens. In Figure 10, the percentage of MIN cells that exhibit this trait is illustrated as an increasing function of age; the remaining cells of the percentage are linear. Since temporal lability was observed sometimes, we counted any cell which displayed a doubling response during any part of the testing period as nonlinear, and thus, Figure 10 probably overestimates the percentage of mature nonlinear responses of MIN cells in kittens. This percentage reaches an adult value at about 8 weeks of age. However, even at 8 weeks of age, the nonlinear response is not always mature as demonstrated by both temporal lability and immature spatial resolutions beyond this age (cf., Figs. 2 and 6).

\section{Discussion}

Every parameter which we examined in the development of MIN neurons changes over the first several postnatal months. Before discussing these changes, we will briefly consider some features of adult MIN neurons.

\section{Adults}

\section{Percentage of Y-cells in MIN}

Our results from recording in MIN of adult cats generally agree with previous studies (Palmer et al., 1975; Mason, 1975; Kratz et al., 1978a, b; Dreher and Sefton, 1979) that have shown this nucleus to be composed almost completely of Y-cells (84 to 100\%). However, as Dreher and Sefton (1979) first noted, an occasional MIN cell $(\simeq 10 \%)$ that had W-or X-cell properties was encountered. It is possible that an electrode sampling bias favoring the large $\mathrm{Y}$-cell somata causes a major $\mathrm{W}$ - or $\mathrm{X}$ cell population to be missed. For instance, a recent anatomical study by Itoh et al. (1981) indicates that many small retinal ganglion cells project to MIN. After injections of horseradish peroxidase into MIN, these authors observed that both large and small ganglion cells were filled retrogradely in the retina. These anatomical findings and the electrophysiological recordings of cells other than Y-cells in MIN directly relate to the interpretation of our results in this paper since we have assumed that MIN is comprised of a nearly pure $(\approx 90 \%)$ Y-cell population.

For threc reasons, we believe that MIN is indeed comprised mainly of Y-cells. First, it is possible, and perhaps likely, that the narrow "wing" of MIN (described by Guillery et al., 1980, as made up of small cells) consists entirely or mostly of W-cells. However, we would have recorded only rarely, if at all, from this area both because of its small size and its dorsal and anterior location relative to most of our penetrations. The vast majority of the remaining MIN neurons would be the Y-cells that we recorded. Second, Itoh et al. (1981) made no attempt to interpret their data in terms of the percentage of $\mathrm{W}$ cells in MIN, but there are at least several reasons why such an interpretation is not presently straightforward. For instance, many of the small ganglion cells that project to the "wing" might have become labeled by the injections. Also, evidence from the A laminae (Friedlander et al., 1981) suggests that large differences exist between ratios of cell types in the retina and lateral geniculate nucleus because of differential divergence in their retinogeniculate projections. Indeed, Y-cells seem relatively more abundant in the A laminae than in the retina and the same might be true for MIN Y-cells. Third, an electrode sampling bias is unlikely to be a significant factor since we routinely recorded from $\mathrm{W}$-cells in the $\mathrm{C}$ laminae with our relatively high impedance electrodes (10 to 30 megohms) and should have sampled a major population of W-cells in MIN if such a population were present. Consequently, we feel that our adult MIN data, 

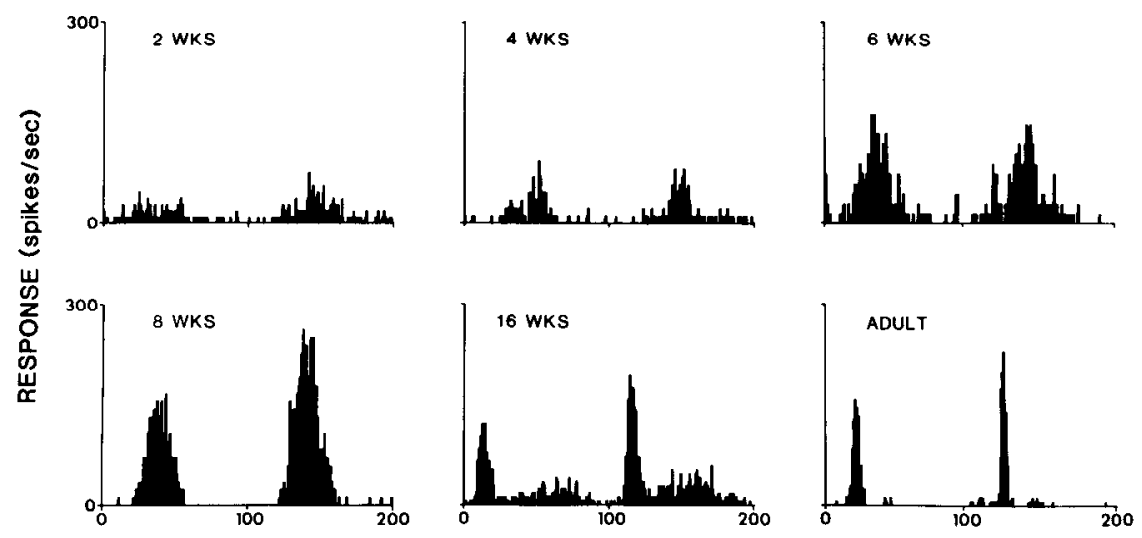

BIN NUMBER

Figure 9. Peristimulus time histograms for 8 different $Y$-cells recorded in MIN of kittens at the ages shown. The stimulus for each was placed in the "null" position for the linear response component. 'These histograms represent typical doubling responses (the best responses that we could obtain for each neuron) for MIN neurons at these ages and demonstrate that these neurons exhibit better nonlinear responses as they mature. However, the widths (time extent of the response) were variable for different neurons at all ages. The stimulus parameters were: spatial frequency, 0.2 cycle/deg for 2 and 16 weeks, 0.1 cycle/deg for 4 weeks, and 0.5 cycle/deg for 6 weeks, 8 weeks, and adult; temporal frequency, 2 $\mathrm{Hz}$ except at 2 weeks which was $1 \mathrm{~Hz}$; spatial and temporal modulation, sine wave for 4 weeks, 16 weeks, and adult and square wave for 2,6, and 8 weeks; contrast, maximum (0.85) in all cases. Fifty trials were taken for all of these neurons except the 2-week example which had 30 trials. Other conventions are as in Figure 2.

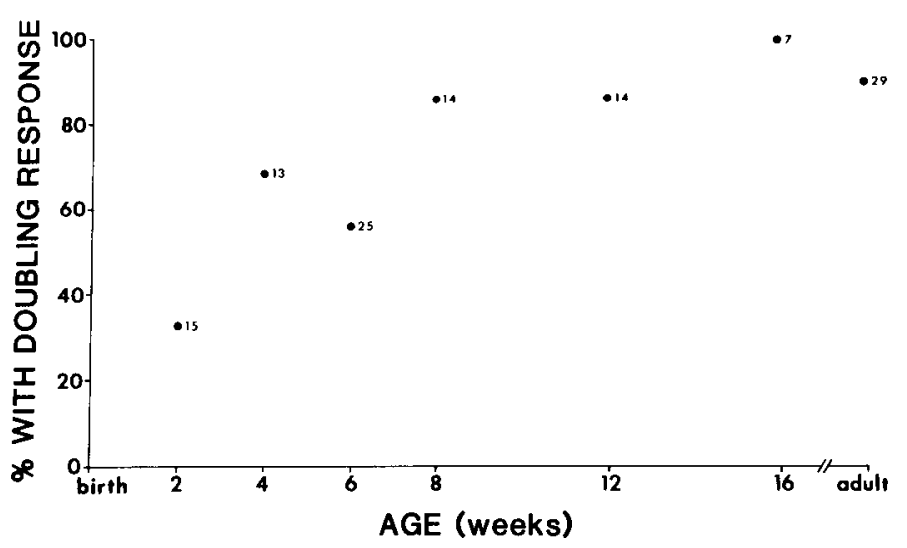

Figure 10. Percentage of the total number of cells recorded in MIN of kittens at different ages which had an audible doubling response to any spatial frequency of a counterphased modulated grating pattern. Virtually all other cells were identified as linear. The total number of cells used to derive this percentage is shown next to each point.

along with the previously cited papers, demonstrate a predominant Y-cell population in MIN.

\section{Receptive field center size}

Our data from adults indicate that there is an increasing receptive field center size with greater visual field eccentricity. This result is intermediate between the observations found by Kratz et al. (1978a) and Dreher and Sefton (1979). The former authors found a very large increase of receptive field size with increasing eccentricity, whereas the latter authors saw no change of receptive field size with eccentricity. Further quantitative studies will be necessary to address this issue more clearly.

\section{Differences between Y-cells of MIN and other geniculate areas}

Most of the characteristics of Y-cells in laminae A, A1, and $\mathrm{C}$ are also common to the Y-cells of MIN. This is not surprising considering that most neurons of MIN receive collaterals from the axons of retinal Y-cells going to these other layers (Bowling and Michael, 1980; Sur and Sherman, 1982b), and the receptive field properties of geniculate cells are almost all derived from their retinal inputs (e.g., Bullier and Norton, 1979; So and Shapley, 1981). Exceptions to the traits common to the cells of MIN and the A laminae are (1) that MIN Y-cells lack the nondominant eye inhibition which the A laminae Ycells usually possess (Kratz et al., 1978a) and (2) MIN cells are not located in close proximity to numerous $\mathrm{X}$ or W-cells unlike Y-cells in the A or C laminae. Two other features seen in this study which appear to distinguish MIN Y-cells from those in the A laminae (but not necessarily those in the $\mathrm{C}$ laminae) are their higher maximum contrast sensitivities and lower spatial resolutions (see "Results"). However, comparisons of this nature may not be appropriate since they are made between two separate studies (Lehmkuhle et al., 1980a, versus the present paper). Further quantitative comparisons of thresholds within one study will be necessary to clarify these points. Qualitatively, we observed (after recording from hundreds of A laminae Y-cells previously) that many MIN Y-cells seem to be more sensitive to contrast and have lower spatial resolutions than do A laminae Ycells. These higher contrast sensitivities and lower spatial resolutions of such MIN Y-cells, coupled with the widespread cortical distribution, suggest that this nucleus serves a more important role in the processing of low 
contrast and low spatial frequency visual information than previously suspected.

\section{Kitten Development}

The postnatal changes in the properties of MIN neurons were mostly as one might expect in a developing sensory system except perhaps for the relatively extended period before certain adult values are reached. That is, MIN neurons, on average, develop their characteristics gradually over the first few months postnatally. We have no data for the range of ages between 4 months and adult, but at 4 months of age, some properties of MIN neurons are not completely mature. A crude extrapolation of our data suggests that complete maturity is achieved at about 6 months of age. Since these data do not derive from individual neurons followed longitudinally, we cannot unambiguously distinguish the actual time course of their development. However, we observed that no kittens of intermediate age (e.g., 8 weeks of age) had some very immature neurons mixed with fully mature ones, even though not all of the cells appeared to be at the same point of development.

\section{Optical aberrations}

A confounding feature in studying the development of MIN neurons is that the optical aberrations in the kitten's eye might be a significant factor in determining the receptive field properties of 2- and 4-week-old kittens (including lack of a doubling response since this property may be attributed to small subfields of the Y-cells; Hochstein and Shapley, 1976). However, by 6 weeks of age, this problem can no longer account for immature properties (Bonds and Freeman, 1978; Freeman and Lai, 1978), including large receptive field center size, lack of a second harmonic, and low temporal and spatial resolutions. Since there are many small receptive fields and reasonable spatial resolutions $(>0.8 \mathrm{cycle} / \mathrm{deg}$ ) for the neurons even in very young animals, this also implies that it is not optical aberrations that cause the large receptive fields and poor spatial resolutions for MIN neurons (see also below). Therefore, these properties are not immature due to poor optics but probably because the neuronal connections and cells themselves are still undeveloped.

\section{Percentage of Y-cells in kitten MIN}

While it is relatively clear that Y-cells make up the vast majority of MIN cells recorded electrophysiologically in the adult (see above), this point is less certain in young kittens due to the difficulty of physiologically identifying and classifying these cells. That is, many of the receptive field properties and latencies were similar in the A laminae, $\mathrm{C}$ laminae, and MIN so that a clear decision could not always be made as to the type of cell being recorded.

We cannot rule out the possibility that there is a significant population of cells within MIN which are not Y-cells and which develop their response properties much earlier than do Y-cells. That is, W-cells might mature before Y-cells in MIN, and these W-cells might be sampled more readily in younger kittens. Our data across the various ages then could reflect a change in relative sampling of two different populations. We think that this is unlikely since our recordings in the A laminae (where there are essentially no W-cells) were unmistakably from some cells (presumably Y-cells due to their doubling responses to grating stimuli) with properties very similar to those seen in MIN. Because immature Ycells with properties similar to those in MIN could be recorded readily elsewhere and they form the major cell type in adult MIN, it also seems likely that the vast majority of cells sampled in kittens were immature Ycells rather than W-cells.

We reiterate that we cannot yet resolve this issue unambiguously. However, even if MIN Y-cells were so immature that we could not sample them in young kittens, our conclusions concerning the late maturation of these Y-cells would still be valid.

\section{Time course and maturation of development}

Latency. The change in latency to optic chiasm stimulation appears to be an exponentially decreasing function that reaches the average adult value at about 12 wecks of age. This is also the time of completion of myelination (Moore et al., 1976). The time courses of the other parameters are much less definitive from our data, but they do represent monotonic and statistically significant changes.

Receptive field center diameter. 'The adult value for receptive field center size appears to be reached between 12 and 16 weeks of age, at which time the mean center diameter is approximately one-third of the value at 2 weeks of age. However, the postnatal enlargement of the kitten's eye during this period produces a decrease in visual angle per unit length of retina such that a decrease of receptive field size by a factor of 1.7 might be expected solely due to this growth of the eye (Olson and Freeman, 1980; see also Hamasaki and Sutija, 1979). This is not sufficient to account completely for the decrease in receptive field diameter that we found and implies an additional neuronal cause for this decrease. Unfortunately, the nature of this neuronal processing cannot be assessed by our data.

Spatial and temporal resolutions. MIN neurons do not appear to reach adult values for spatial and temporal resolution by 16 weeks of age (this being the oldest kitten from which we recorded). This is beyond the period where most of the neuronal properties are adult-like (including the number of cells having doubling responses; see also Mangel et al., 1980). Y-cells in MIN thus appear to develop some properties throughout the first 4 to 6 months of age. This long development time might influence these properties more than the other receptive field properties when the kitten is visually deprived by lid suture or dark rearing (see below and Daniels et al., 1978). Visual acuity, as measured by behavioral methods (Mitchell et al., 1976), exceeds the low spatial resolution of most of the MIN neurons (adult and kitten), and it would seem unlikely that MIN contributes much to this parameter.

Nonlinear responses. Hochstein and Shapley (1976) postulated that small subunits account for the nonlinearities or doubling responses of Y-cells. If this is the case, then our data indicate that these subunits develop later 
than do the linear response components. Regardless of the nature of the underlying mechanisms of doubling, it is not until 8 weeks of age that the adult percentage of $\mathrm{Y}$-cells with doubling responses is reached, and, therefore, this feature is not always a reliable indicator of an inmature $\mathrm{Y}$-cell before this age. Even at 8 weeks of age, the development of the nonlinear response component is not complete since some MIN cells still show variable amplitudes of this response and MIN spatial resolution development continues beyond 16 weeks of age. Furthermore, there are cells with on/off centers in immature animals both in MIN and the A laminae (this study; Daniels et al., 1978), and it is not yet known if these all develop into $\mathrm{Y}$-cells.

\section{Comparisons with other data}

Daniels et al. (1978) followed the development of cells in the A laminae of the lateral geniculate nucleus from birth to 7 weeks of age. Since the results are fairly consistent between their study and our own (considering that we looked mostly at MIN Y-cells and only a small number of A laminae cells), we have only a few comments in our comparison. First, we observe that it is not until 8 weeks of age that the adult proportion of MIN neurons which show a second harmonic or doubling response component is reached. Because there are so many similarities between Y-cells in MIN and the A laminae, it is likely that there are many immature $\mathrm{Y}$-cells in the $\mathrm{A}$ laminae of young kittens ( $<8$ weeks) which also lack nonlinearities. Without other clear features to identify and classify the cells in these laminae, it is possible that some cells may have been misclassified by these authors (see Fig. 9). Second, although our sample of cells in the A laminae is quite small, we saw many examples of neurons which had mature or nearly mature properties indicative of X-cells, while MIN neurons sampled in the same cats still had many immature properties (large receptive fields, poor temporal resolutions, etc.). Thus, we concur with Daniels et al. (1978) that X-cells can achieve maturity of many of their receptive properties prior to those of Y-cells, but one qualification must be considered for this last statement. The spatial resolutions of X-cells appear to require a considerably longer period of time to develop (24 weeks or more; Mangel et al., 1980) relative to the other cell characteristics. The term mature or adult neuron implies that it is fully developed in all respects so that most $\mathrm{X}$-cells require about 24 weeks to develop completely. Y-cells also appear to require about this same time period for maturation of their spatial and temporal resolutions. Thus, long or late periods of development may relate to the adverse effects of lid suture on many features of $\mathrm{Y}$-cells but only on the spatial resolution of X-cells (Lehmkuhle et al., 1978, 1980b; Mangel et al., 1980).

\section{Immature and deprived MIN neurons}

Monocular deprivation severely affects the neurons in MIN which receive input from the deprived eye. Kratz et al. (1978b) showed both electrophysiological and morphological changes in MIN after such deprivation. Fewer cells could be recorded from the deprived MIN area (i.e., receiving retinal input from the deprived eye) than from the nondeprived or normal areas, and many of the deprived cells which were active had abnormal characteristics. These abnormalities included (1) long latencies to optic chiasm stimulation, (2) poor or inconsistent responses to visual stimuli, (3) large receptive field center sizes, (4) on/off center responses to flashed spots of light, (5) lack of response to fast moving discs, and (6) tonic responses to standing contrast. Also, nearly all (9 out of 12) of these abnormal cells had "null" positions to a counterphased modulated grating instead of the normal doubling responses of Y-cells (K. E. Kratz, S. V. Webb, and S. M. Sherman, unpublished observations). All of these abnormal properties, except tonic responses, are much like the properties that we observed for immature Y-cells in MIN. This close similarity suggests a lack of development of many deprived Y-cells during monocular deprivation.

\section{Summary}

MIN Y-cells in 2-week-old kittens are immature in terms of their latencies to optic chiasm stimulation and receptive field properties. The average latencies to optic chiasm stimulation and receptive field center size reach maturity at about 3 to 4 months. Spatial and temporal resolutions and the nonlinear response properties are the last properties to reach adult values and they do so at approximately 4 to 6 months of age, although most MIN neurons exhibit mature nonlinear responses by 8 weeks of age. A comparison of these immature Y-cell properties with adult, visually deprived $\mathrm{Y}$-cells of MIN revealed a close similarity between the two populations. This result is consistent with the idea that monocular visual deprivation causes a cessation of development of these neurons.

\section{References}

Bonds, A. B., and R. D. Freeman (1978) Development of optical quality in the kitten eye. Vision Res. 18: 391-398.

Bowling, D. B., and C. R. Michael (1980) Projection patterns of single physiologically characterized optic tract fibres in cat. Nature 286: 899-902.

Bullier, J., and T. T. Norton (1979) Comparison of receptive field properties of $\mathrm{X}$ and $\mathrm{Y}$ ganglion cells with $\mathrm{X}$ and $\mathrm{Y}$ geniculate cells in the cat. J. Neurophysiol. 42: 274-291.

Daniels, J. D., J. D. Pettigrew, and J. L. Norman (1978) Development of single-neuron responses in kitten's lateral geniculate nucleus. J. Neurophysiol. 41: 1373-1393.

Dreher, B., and A. J. Sefton (1979) Properties of neurons in cat's dorsal lateral geniculate nucleus: A comparison between medial interlaminar and laminated parts of the nucleus. J. Comp. Neurol. 183: 47-64.

Enroth-Cugell, C., and J. G. Robson (1966) The contrast sensitivity of retinal ganglion cells of the cat. J. Physiol. (Lond.) 187: 517-552.

Fernald, R., and R. Chase (1971) An improved method for plotting retinal landmarks and focusing the eyes. Vision Res. 11: 95-96.

Freeman, R. D. (1980) Corneal radius of curvature of the kitten and the cat. Invest. Ophthalmol. Vis. Sci. 19: 306-308.

Freeman, R. D., and C. E. Lai (1978) Development of the optical surfaces of the kitten eye. Vision Res. 18: 399-407.

Friedlander, M. J., C. -S. Lin, L. R. Stanford, and S. M. Sherman (1981) Morphology of functionally identified neurons in lateral geniculate nucleus of the cat. J. Neurophysiol. 46: $80-129$. 
Garey, L. J., and T. P. S. Powell (1967) The projection of the lateral geniculate nucleus upon the cortex in the cat. Proc. R. Soc. Lond. (Biol.) 169: 107-126.

Garey, L. J., and T. P. S. Powell (1968) The projection of the retina in the cat. J. Anat. 102: 189-222.

Geisert, E. E. (1980) Cortical projections of the lateral geniculate nucleus in the cat. J. Comp. Neurol. 190: 793-812.

Gilbert, C. D., and J. P. Kelly (1975) The projections of cells in different layers of the cat's visual cortex. J. Comp. Neurol. 163: 81-106.

Guillery, R. W. (1970) The laminar distribution of retinal fibers in the dorsal lateral geniculate nucleus of the cat: $A$ new interpretation. J. Comp. Neurol. 138: 339-368.

Guillery, R. W., E. E. Geisert, E. H. Polley, and C. A. Mason (1980) An analysis of the retinal afferents to the cat's medial interlaminar nucleus and to its rostral thalamic extension, the "geniculate wing." J. Comp. Neurol. 194: 117-142.

Hamasaki, D. I., and V. G. Sutija (1979) Development of Xand Y-cells in kittens. Exp. Brain Res. 35: 9-23.

Hammond, P., and C. R. James (1971) The Purkinje shift in cat: Extent of the mesopic range. J. Physiol. (Lond.) 216: 99-109.

Hochstein, S., and R. M. Shapley (1976) Quantitative analysis of retinal ganglion cell classifications. J. Physiol. (Lond.) 262: 237-264.

Hollander, H., and J. Vanegas (1977) The projection from the lateral geniculate nucleus onto the visual cortex in the cat. A quantitative study with horseradish peroxidase. J. Comp. Neurol. 173: 519-536.

Itoh, K., M. Conley, and I. T. Diamond (1981) Different distributions of large and small retinal ganglion cells in the cat after HRP injections of single layers of the lateral geniculate body and the superior colliculus. Brain Res. 207: 147-152.

Karamanlidis, A. N., and J. Magras (1972) Retinal projections in domestic ungulates. I. The retinal projections in the sheep and the pig. Brain Res. 44: 127-145.

Karamanlidis, A. N., and J. Magras (1974) Retinal projections in domestic ungulates. II. The retinal projections in the horse and the ox. Brain Res. 66: 209-225.

Kratz, K. E., S. V. Webb, and S. M. Sherman (1978a) Studies of the cat's medial interlaminar nucleus: A subdivision of the dorsal lateral geniculate nucleus. J. Comp. Neurol. 181: 601-614.

Kratz, K. E., S. V. Webb, and S. M. Sherman (1978b) Effects of early monocular lid-suture upon neurons in the cat's medial interlaminar nucleus. J. Comp. Neurol. 181: 615-626.

Lehmkuhle, S. W., K. E. Kratz, S. Mangel, and S. M. Sherman (1978) An effect of early monocular lid suture upon the development of $\mathrm{X}$-cells in the cat's lateral geniculate nucleus. Brain Res. 157: 346-350.

Lehmkuhle, S. W., K. E. Kratz, S. Mangel, and S. M. Sherman (1980a) Spatial and temporal sensitivity of X-and Y-cells in the dorsal lateral geniculate nucleus of the cat. J. Neurophysiol. 43: 520-541.

Lehmkuhle, S. W., K. E. Kratz, S. Mangel, and S. M. Sherman (1980b) Effects of early monocular lid suture on spatial and temporal sensitivity of neurons in dorsal lateral geniculate nucleus of the cat. J. Neurophysiol. 43: 542-556.

LeVay, S., and D. Ferster (1977) Relay cell classes in the lateral geniculate nucleus of the cat and the effects of visual deprivation. J. Comp. Neurol. 172: 563-584.

Maciewicz, R. J. (1974) Afferents to the lateral suprasylvian gyrus of the cat traced with horseradish peroxidase. Brain Res. 78: 139-143.

Maciewicz, R. J. (1975) Thalamic afferents to 17, 18, and 19 of cat cortex traced with horseradish peroxidase. Brain Res. 84: 308-312.
Mangel, S., J. R. Wilson, and S. M. Sherman (1980) Development of neuronal response properties in the cat lateral geniculate during monocular lid-suture. Soc. Neurosci. Abstr. 6: 584.

Mason, R. (1975) Cell properties in the medial interlaminar nucleus of the cat's lateral geniculate complex in relation to the transient/sustained classification. Exp. Brain Res. 22: 327-329.

Mitchell, D. E., F. Griffin, F. Wilkerson, P. Anderson, and M. L. Smith (1976) Visual resolution in young kittens. Vision Res. 16: 363-366.

Moore, C. L., R. Kalil, and W. Richards (1976) Development of myelination in optic tract of the cat. J. Comp. Neurol. 165: 125-136.

Movshon, J. A., and R. C. Van Sluyters (1981) Visual neural development. Annu. Rev. Psychol. 32: 477-522.

Niimi, K., and J. M. Sprague (1970) Thalamo-cortical organization of the visual system in the cat. J. Comp. Neurol. 138: 219-250.

Olson, C. R., and R. D. Freeman (1980) Rescaling of the retinal map of visual space during growth of the kitten's eye. Brain Res. 186: 55-65.

Palmer, L. A., A. C. Rosenquist, and R. Tusa (1975) Visual receptive fields in lam LGNd, MIN, and PN of the cat. Soc. Neurosci. Abstr. 1: 54 .

Rosenquist, A. C., S. B. Edwards, and L. A. Palmer (1974) An autoradiographic study of the projections of the dorsal lateral geniculate nucleus and posterior nucleus in the cat. Brain Res. 80: 71-93.

Sanderson, K. J. (1971) The projection of the visual field to the lateral geniculate and medial interlaminar nuclei in the cat. J. Comp. Neurol. 143: 101-118.

Sanderson, K. J. (1974) Lamination of the dorsal lateral geniculate nucleus in carnivores of the weasel (Mustelidae), raccoon (Procyonidae) and fox (Canidae) families. J. Comp. Neurol. 153: 239-266.

Sherman, S. M., and P. D. Spear (1982) Organization of the visual pathways in normal and visually deprived cats. Physiol. Rev., in press.

Siegel, S. (1956) Nonparametric Statistics for the Behavioral Sciences, pp. 184-193, McGraw-Hill Publications, New York. So, Y. T., and R. Shapley (1981) Spatial tuning of cells in and around lateral geniculate nucleus of the cat: $\mathrm{X}$ and $\mathrm{Y}$ relay cells and perigeniculate interneurons. J. Neurophysiol. 45: 107-120.

Stanford, L. R., M. J. Friedlander, and S. M. Sherman (1981) Morphology of physiologically identified $W$-cells in the $C$ laminae of the cat's lateral geniculate nucleus. J. Neurosci. 1: 578-584.

Sur, M., and S. M. Sherman (1982a) Linear and nonlinear Wcells in the C laminae of the cat's lateral geniculate nucleus. .J. Neurophysiol., in press.

Sur, M., and S. M. Sherman (1982b) Retinogeniculate terminations in cats: Morphological differences between X-and Ycell axons. Science, in press.

Thorn, F., M. Gollender, and P. Erickson (1976) The development of the kitten's visual optics. Vision Res. 16: 1145-1149.

Thuma, B. D. (1928) Studies of the diencephalon of the cat. 1. The cyto-architecture of the corpus geniculate laterale. J. Comp. Neurol. 46: 173-199.

Updyke, B. V. (1975) The patterns of projections of cortical areas 17,18 , and 19 onto the laminae of the dorsal lateral geniculate nucleus in the cat. J. Comp. Neurol. 163: 377-395.

Wilson, P. D., M. H. Rowe, and J. Stone (1976) Properties of relay cells in cat's lateral geniculate nucleus: A comparison of W-cells with X- and Y-cells. J. Neurophysiol. 39: 1193-1207. 